\title{
The intercontinental schizophrenia outpatient health outcomes (ic-soho) study : baseline clinical and functional characteristics and antipsychotic use patterns in the North Africa and Middle Eastern (amea) region
}

\author{
Frans A. Korb ${ }^{1}$, Çınar Yenilmez ${ }^{2}$, Abderrahmane Belaid ${ }^{3}$, Mohamed Ghazi ${ }^{4}$, Abdel-Naser Omar ${ }^{5}$, Istvan Bitter ${ }^{6}$ \\ 'Clinical Research Physician Neuroscience, Eli Lilly Regional Operations, Vienna, Austria, Osmangazi University Medical Faculty, ${ }_{5}^{2}$ sychiatry \\ Department, Me_elik, Eski_ehir-Turkey, EHS Psychiatrie Cheraga, Alger, Algeria, Al-Manaa Hospiatal , Dammam, Saudi Arabia, Ain Shams \\ University, Cairo, Egypt, Senior Clinical Research Physician \& Head of Neuroscience, Eli Lilly Regional Operations, Vienna, Austria
}

\begin{abstract}
Objective: To describe the baseline findings of the Intercontinental Schizophrenia Outpatient Health Outcomes (IC-SOHO) study in the North Africa and Middle Eastern sub-region (AMEA-SOHO) Method: The IC-SOHO study is an ongoing prospective, three-year, non-interventional observational study of schizophrenia treatment, clinical characteristics and mental health services utilization in two North African and two Middle Eastern countries. The study population consists of non-hospitalised patients who had initiated treatment with or changed to a new antipsychotic. Results: The baseline findings of the IC-SOHO study (AMEA Subset) appear to reflect clinical practice in Turkey, Saudi Arabia, Egypt and Algeria $(N=1,398)$. Overall, the patients were moderately to markedly ill and either overweight (46\%) or obese (8\%) when they entered the study. Functionally, the majority of patients were not involved in social activities, could not care for themselves and were unemployed. Substance and alcohol dependency/abuse was not a problem in this study population. At baseline the majority of patients were treated with typical antipsychotics (oral and depot); and anticholinergics were the most commonly prescribed concomitant medication. Sexual side effects were most frequently reported among the surveyed adverse events. Overall complianceladherence to medication was good. Conclusion: The baseline IC-SOHO data highlighted various clinical and functional characteristics and antipsychotic use patterns in a group of outpatients with schizophrenia in a naturalistic setting. Once completed, the IC-SOHO study will add further to this knowledge base.
\end{abstract}

Key words: Schizophrenia, Outpatients, Antipsychotic agents, Clinical characteristics, Functional characteristics, North Africa, Middle East, IC-SOHO

This paper is one of two similar papers reporting aspects of the Intercontinental Schizophrenia Outpatient Health Outcomes (IC-SOHO) Study. The other 'Intercontinental Schizophrenia Outpatient Health Outcomes (IC-SOHO) Study: Baseline Clinical and Functional Characteristics and Antipsychotic Use Patterns in the Central and Eastern Europe (CEE) Region' has been submitted for publication to Neuropsychiatrie.

\section{Correspondence:}

Dr FA Korb, Eli Lilly (SA), PBag X119, Bryanston,2021, Johannesburg, South Africa

email:korb_frans@lilly.com
The emphasis in evaluating drug treatment benefits is shifting from 'efficacy' to 'effectiveness'. Efficacy is defined as the desirable effect of an intervention, whereas effectiveness is the extent to which a product works in the patients to whom it has been offered. This meaning is slightly different from 'efficacy', which can be measured in those who have actually been treated. 'Efficacy' relates to explanatory studies and 'effectiveness' to pragmatic studies. ${ }^{1}$ Therapeutic efficacy (effectiveness) is often studied using observational surveys of patients whose treatments were selected nonexperimentally. New methodological strategies are being developed that may improve the quality of observational studies. These are, often based on the design principles and patient assembly procedures of the randomized trial. ${ }^{2}$

Randomized clinical trials (RCTs) have been considered the 'gold standard' for establishing safety and efficacy due to 
their strong internal validity. Given a set of assumptions, RCTs are designed to achieve a valid comparison of alternative treatments. The process of randomization reduces bias in order to compare and equalize treatment and control groups with respect to all variables except the assignment of treatment. In this way, a causal relationship between treatment and outcome can be established. RCTs are usually conducted applying strict protocol and regulatory guidelines. For these reasons, RCTs have been criticized for lacking external validity. This implies that results obtained from RCTs can often not be generalized to the actual affects seen when the drug is used in clinical practice.

RCTs have long been the cornerstone of pharmaceutical drug development as well as the eventual drug registration and regulation process. The demand for new methods to monitor drug safety, efficacy and effectiveness has risen to the extent that the discontinuation of phases III and IV of placebo controlled trials and the enhancement of the use of post-marketing surveillance have been suggested. When a drug is commercialised, the question changes from one of safety and efficacy ('can it work'?) to one of effectiveness ('does it work'?). , $^{3,4,5}$

Observational studies examine natural variations in exposure to treatments and describe associated outcomes. ${ }^{1}$ The major criticism of observational studies is linked to potential allocation bias and risk adjustment. ${ }^{5}$ In order to account for these factors, observational studies can be designed with rigorous methodology and statistical methods that mimic those of clinical trials. Recent studies conducted across multiple therapeutic areas comparing RCTs and observational study findings found no major differences among estimates of treatment effects. ${ }^{6,7,8,9,10,11,12,13,14}$ In conclusion, both RCTs and nonrandomized (observational) studies can provide complimentary evidence.

A growing number of observational studies have been published which assess the treatment effects of atypical antipsychotics in schizophrenia: clozapine, sertindole, risperidone and olanzapine. ${ }^{15,16,17,18,19,20,21,22}$ Brambilla et al ${ }^{15} \mathrm{re}-$ viewed 50 experimental and observational studies evaluating clozapine-treated subjects in treatment-resistant schizophrenia. They reported that most studies enrolled a small sample of patients (less than 50) and were retrospective in design. Overall, the vast majority of RCTs and observational studies (both prospective and retrospective in design) have had a short duration (less than 8 and 12 weeks respectively). Observational trials also adopted implicit criteria for patient selection.

New observational studies are thus needed which incorporate a larger sample group, are prospective in design, longer in duration and apply explicit selection criteria. The Intercontinental Schizophrenia Outpatient Health Outcomes (ICSOHO) study was initiated to address this need. The ICSOHO study was designed to assess antipsychotic medication therapy outcomes for schizophrenia patients in actual clinical practice. This paper reports the IC-SOHO study baseline findings in the North African and Middle Eastern region (AMEA). The primary objective of the analyses reported here was to determine the antipsychotic use patterns of schizophrenia patients in an outpatient setting. In addition, the baseline clinical and functional characteristics were examined in these patients.

\section{Material and Methods}

\section{Study Design and Patients}

The IC-SOHO study is a longitudinal (3-year), noninterventional, prospective, observational, open-label study of the treatment of schizophrenia in two North African and two Middle Eastern countries. The primary objective of the study is to assess the costs and outcomes of antipsychotic treatment of schizophrenia using antipsychotics, with specific focus on the atypical antipsychotic,olanzapine. The secondary objectives of the study is to increase understanding of pharmacological treatment patterns for schizophrenia. The ICSOHO study is currently being conducted in 27 countries around the world. This article reports on data collected from Algeria, Egypt, Saudi Arabia and Turkey.

Participating psychiatrists offered enrolment on a discretionary basis to patients who met the following criteria :-

i. present within the current course of care; ii. will initiate or change antipsychotic medication for the treatment of schizophrenia (ICD-10 or DSM-IV Criteria); iii. will initiate or change antipsychotic medication in the outpatient setting or in the hospital when the admission was planned for the antipsychotic initiation or change; iv. at least 18 years of age, and $\mathrm{v}$. will not be simultaneously participating in a different study that includes a treatment intervention and/or an investigational drug. Patients were included regardless of whether the new antipsychotic drug substituted a previous medication or was an addition to existing treatment, and regardless of the reason for the treatment change. Newly diagnosed and neuroleptic-naïve patients were included in the study. Depending on the local regulations in the participating countries, all patients provided at least oral consent to participate in the study.

The IC-SOHO study was designed to provide two patient cohorts of approximately equal size: a first group of patients who were initiated or changed to olanzapine therapy, and a second group who were initiated or changed to non-olanzapine antipsychotic therapy.

Each participating psychiatrist was requested to enroll at least one block of ten patients, with five patients in each cohort. This was done by allocating alternate patients to each cohort in order to provide approximately equal numbers in each cohort. Investigators were instructed to make treatment decisions independent of the study, and then to evaluate whether patients were eligible for inclusion based on the entry criteria and the alternating structure of enrolment.

Data were collected on a standardized collection form which took approximately 15 to 20 minutes to complete. The data collected were similar to those usually collected in routine clinical practice, including patient demographics, medical resource use, clinical, functional and social status, antipsychotic and other co-prescribed medication use, tolerability, patient- and physician-reported compliance, sexual function, alcohol and substance abuse, and quality of life. Clinical status was assessed using the Clinical Global Impression - Severity (CGI-S) scale (24). For the purpose of this study, the symptom domains related to schizophrenia measured on the CGI-S scale included overall symptom severity, positive symptoms, negative symptoms, depressive symptoms and cognitive symptoms. Health-related Quality of Life was assessed using the EuroQol (EQ-5D) scale consisting of five items and the Visual Analogue Scale. ${ }^{23,24}$ 


\section{Statistical Analysis}

The first step in the evaluation of the study data was to use exploratory and descriptive analyses to gain an understanding of the qualitative and quantitative nature of the data collected and thus the characteristics of the sample studied. This manuscript reports the observational data recorded at the first (baseline) visit. Data collected at baseline included patient characteristics described by socio-demographic, clinical and functional status for the total group of patients as well as by gender. Baseline data for all the groups and parameters were compared through the calculation of percentages, means and standard deviations. For the symptom CGI-Severity items a Cochran-Mantel-Haenszel test was used to calculate differences between the categorical variables (Table 2)

\section{Results}

A total of 7,648 patients were enrolled in the IC-SOHO study at baseline. Enrollment took place for a twelve-month period from December 2000 to November 2001. Four countries in the AMEA region contributed 1,398 patients to the study.

\begin{tabular}{|ll|}
\hline TABLE 1: Patient Characteristics (AMEA subset) \\
\hline Characteristic \\
\hline Number of patients enrolled (\%) \\
Gender (\%) male & $1,398(100)$ \\
female & $913(66)$ \\
Mean age (SD) & $479(34)$ \\
Percent of patients neuroleptic-naive & $32.5(10.12)$ \\
CGI overall symptoms: mean (SD) & 18.3 \\
CGI positive symptoms: mean (SD) & $3.6(1.03)$ \\
CGI negative symptoms: mean (SD) & $3.4(1.38)$ \\
CGI cognitive symptoms: mean (SD) & $3.1(1.37)$ \\
CGI depressive symptoms: mean (SD) & $2.8(1.36)$ \\
Patients who have attempted suicide: (\%) (n=160): & $2.3(1.42)$ \\
Ever (\%) & 13.1 \\
Subjects with suicide attempts in the previous 6 months (\%) & 7.8 \\
\hline
\end{tabular}

Those were Turkey with $692(49.5 \%)$ patients, Algeria with $306(22.9 \%)$ patients, Saudi Arabia with 204 (14.6\%) patients and Egypt with 196 (14\%) patients. The study population consisted of $913(66 \%)$ male and $479(34 \%)$ female patients with a mean age of 32.5 years (Table 1 ).

\section{Clinical Characteristics}

The CGI-Severity scores range from 1 to 7 , with 1 being 'nor$\mathrm{mal} / \mathrm{not}$ at all ill', and 7 'among the most severely ill'. The CGI-S overall mean score was 3.6 (moderately to markedly ill)(Table 1). At baseline there appears to be a tendency for males to be more moderately ill while the females tended to be more markedly ill. However, this was not statistically significant (Table 2).

Symptom severity (CGI-S) was also individually measured for the four symptom clusters associated with schizophrenia. At baseline, patients presented mostly with positive and negative symptoms (Table 1). Females tended to have slightly more positive symptoms (marked and worse) than the male group, and males presented with more negative symptoms (mild and worse) (Table 2). Cognitive symptoms were slightly less severe with depressive symptoms the least severe (Table 1). No statistical difference was found between cognitive (male and female groups) and depressive (male and female groups) (Table 2). A significant proportion of patients reported that they had attempted suicide in the past, with a smaller percentage of the attempts being in the last 6 months prior to the baseline visit (Table 1).

\section{Functional Characteristics}

In terms of functional status, a third of patients were involved in a relationship (married or in a permanent relationship) with a spouse or partner, almost half were socially active. Convesrsely, more than half of patients had engaged in no social activities with friends or family in the 4 weeks prior to the study commencing (Table 3 ). Approximately a quarter of the AMEA-SOHO patients could care for themselves in inde-

TABLE 2: Symptom CGI-Severity (\%) by Gender (AMEA subset)

\begin{tabular}{|c|c|c|c|c|c|c|c|}
\hline & Normal & Borderline & Mild & Moderate & Marked & Severe & Most Severe \\
\hline $\begin{array}{l}\text { Overall symptoms }(\mathrm{p}=0.4770)^{*} \\
\text { Male } \\
\text { Female }\end{array}$ & $\begin{array}{l}0.2 \\
0.2\end{array}$ & $\begin{array}{l}2.1 \\
3.1\end{array}$ & $\begin{array}{l}9.0 \\
8.4\end{array}$ & $\begin{array}{l}33.7 \\
27.7\end{array}$ & \begin{tabular}{|l|}
36.6 \\
42.8
\end{tabular} & $\begin{array}{l}15.5 \\
14.5\end{array}$ & $\begin{array}{l}2.9 \\
3.4\end{array}$ \\
\hline $\begin{array}{l}\text { Positive symptoms }(p=0.0041)^{*} \\
\text { Male } \\
\text { Female }\end{array}$ & $\begin{array}{l}3.7 \\
2.1\end{array}$ & $\begin{array}{l}7.1 \\
5.9\end{array}$ & $\begin{array}{l}14.6 \\
11.5\end{array}$ & $\begin{array}{l}25.6 \\
25.7\end{array}$ & \begin{tabular}{|l|}
28.6 \\
31.0
\end{tabular} & $\begin{array}{l}16.9 \\
18.2\end{array}$ & $\begin{array}{l}3.4 \\
5.6\end{array}$ \\
\hline $\begin{array}{l}\text { Negative symptoms }(\mathrm{p}=0.0162)^{*} \\
\text { Male } \\
\text { Female }\end{array}$ & $\begin{array}{l}3.2 \\
5.0\end{array}$ & $\begin{array}{l}8.4 \\
12.6\end{array}$ & $\begin{array}{l}17.5 \\
15.8\end{array}$ & $\begin{array}{l}29.4 \\
28.8\end{array}$ & \begin{tabular}{|l|}
26.3 \\
24.6
\end{tabular} & $\begin{array}{l}12.4 \\
11.1\end{array}$ & $\begin{array}{l}2.9 \\
2.1\end{array}$ \\
\hline $\begin{array}{l}\text { Cognitive symptoms }(p=0.2035)^{*} \\
\text { Male } \\
\text { Female }\end{array}$ & $\begin{array}{l}5.7 \\
7.8\end{array}$ & $\begin{array}{l}10.8 \\
13.7\end{array}$ & $\begin{array}{l}22.9 \\
17.8\end{array}$ & $\begin{array}{l}29.0 \\
28.8\end{array}$ & $\begin{array}{l}21.6 \\
24.3\end{array}$ & $\begin{array}{l}8.7 \\
7.0\end{array}$ & $\begin{array}{l}1.3 \\
0.6\end{array}$ \\
\hline $\begin{array}{l}\text { Depressive symptoms }(\mathrm{p}=0.1944)^{*} \\
\text { Male } \\
\text { Female }\end{array}$ & $\begin{array}{l}11.0 \\
14.2\end{array}$ & $\begin{array}{l}19.5 \\
17.8\end{array}$ & $\begin{array}{l}22.6 \\
21.4\end{array}$ & \begin{tabular}{|l}
24.4 \\
28.5
\end{tabular} & $\begin{array}{l}16.7 \\
12.5\end{array}$ & $\begin{array}{l}5.0 \\
5.7\end{array}$ & $\begin{array}{l}0.9 \\
0.0\end{array}$ \\
\hline
\end{tabular}

${ }^{*}$ Cochran Mantel-Haenszel Mean Score Test 


\begin{tabular}{|lll|}
\hline $\begin{array}{l}\text { TABLE 3: Work Status and Social and Living Conditions in the past } 4 \text { weeks } \\
\text { (\%) (AMEA subset) }\end{array}$ \\
\hline Employment: & Employed & 22.5 \\
& Able but no job & 19.5 \\
& Unable & 43.2 \\
\hline \multirow{2}{*}{ Social Status: } & Relationship* & 30.7 \\
& Socially active & 47.2 \\
& No socializing & 52.7 \\
\hline \multirow{2}{*}{ Living Conditions: } & Independent & 24.6 \\
& Dependent & 70.8 \\
& Supervised & 2.4 \\
\hline *Relationship=married or in a permanent relationship \\
\hline
\end{tabular}

pendent living conditions. The other three-quarters of the patients reported that they were in residence as a dependent family member, and a small percentage lived in a supervised residence (Table 3). With regard to employment, just over twenty percent of patients in the AMEA-SOHO study were employed. A lower percentage felt that they were unemployed but available to work. Over forty percent reported that they were unable to work (Table 3).

Patient behavior was also assessed at enrolment into the IC-SOHO study. The vast majority of AMEA patients reported that they had never suffered from or been diagnosed with substance dependency/abuse. A limited number of the patients reported that they had suffered from or been diagnosed with substance dependency/abuse in the past, and an almost negligible number of patients admitted that they currently suffered from or had been diagnosed with substance dependency/abuse (Table 4). A similar pattern was true for alcohol dependency/ abuse. Almost ninety-three percent of patients reported that they had never suffered from or been diagnosed with alcohol dependency/abuse. A limited number of the patients reported that they had suffered from or been diagnosed with alcohol dependency/abuse in the past, and about one percent admitted that they were currently suffering from or had been diagnosed as having alcohol dependency/abuse (Table 4).

Almost a half of the patients reported that they had exhibited verbal or physical hostility/aggression in the past 6 months. A small percentage of the patients had been compulsorily admitted, arrested or had spent at least one night in jail in the past 6 months. A minority of patients had been a victim of violent crime (e.g. robbery, mugging, assault or rape) in the past 6 months (Table 4 ).

\begin{tabular}{|llr|}
\hline TABLE 4: Patient Behavior (\%) (AMEA subset) & \\
\hline Asocial Behavior (Past 6 months): & Arrested/jailed & 10.8 \\
& Verbal/physical aggression & 43.1 \\
& Victimized & 2.8 \\
\hline Substance Abuse: & Never & 93.8 \\
& In the past & 6.2 \\
& Current & 1.4 \\
\hline \multirow{2}{*}{ Alcohol Abuse: } & Never & 92.8 \\
& In the past & 7.2 \\
& Current & 0.9 \\
\hline
\end{tabular}

\begin{tabular}{|c|c|}
\hline TABLE 5: Pattern of Antips & nt Drug \\
\hline Antipsychotic (in the pa & \\
\hline Oral typical & 59.9 \\
\hline Depot typical & 25.1 \\
\hline Oral atypical & 37.2 \\
\hline No antipsychotic & 18.3 \\
\hline Concomitant Drugs (upo & \\
\hline Anticholinergics & 38.6 \\
\hline Antidepressants & 11.7 \\
\hline Anxiolytics/hypnotics & 13.1 \\
\hline Mood stabilizers & 6.7 \\
\hline None & 48.5 \\
\hline
\end{tabular}

Antipsychotic Drug Use

Table 5 summarizes the pattern of antipsychotic (in the past 6 months) and concomitant (upon presentation) drug use. At the AMEA-SOHO baseline visit, prior to the patient initiating or changing their antipsychotic treatment, more than half the patients were being treated with a typical oral antipsychotic. A further quarter of patients were on a depot typical antipsychotic. Conversely more than a third of patients was receiving an oral atypical antipsychotic agent. Almost a fifth of the patients were neuroleptic-naïve.

At enrolment into the study, almost half of the patients were not on any concomitant medication. Anticholinergics were the most common concomitant medication. This was followed by the use of anxiolytics/hypnotics, antidepressants and (to a minimal degree) mood stabilizers (Table 5).

Weight was recorded and body mass index (BMI) was calculated for all patients that entered the study. More than half the patients enrolled in the AMEA-SOHO study were overweight $(45.8 \% ; 24=<\mathrm{BMI}<31)$ or obese $(7.6 \% ; \mathrm{BMI}>=31)$. About forty percent had a normal weight $(41.5 \%$ $; 19=<\mathrm{BMI}<24)$ while only $5.1 \%$ were underweight $(\mathrm{BMI}<19)$.

Historically, evaluations of side effects associated with typical and atypical antipsychotics have included weight, EPS, sexual dysfunction and prolactin elevation. Therefore, these have also been selected and included in this study.

When patients entered the IC-SOHO study, an assessment was made regarding current side effects associated with antipsychotic drug therapy (Table 6). The most frequently reported side effect was that of loss of libido. The incidence of extra-pyramidal symptoms (dystonia/akathisia/parkinsonism) was only slightly lower. This was followed by impotence and

\begin{tabular}{|lc|}
\hline TABLE 6: Adverse Events Reported (\%) (AMEA subset) \\
\hline Loss of libido ( $\mathrm{n}=1371)$ & 43.5 \\
EPS ( $\mathrm{n}=1378)$ & 37.6 \\
Impotence/sexual dysfunction ( $\mathrm{n}=1349)$ & 35.3 \\
Menstrual problems (woman) ( $\mathrm{n}=479)$ & 12.2 \\
Tardive dyskinesia ( $\mathrm{n}=1374)$ & 6.2 \\
Galactorrhoea ( $\mathrm{n}=1299)$ & 3.6 \\
\hline n=patients for whom adverse events were applicable and available
\end{tabular}


sexual dysfunction, menstrual disturbance and amenorrhoea in woman, tardive dyskinesia, with the lowest reported adverse event being galactorrhoea. Some patients reported multiple adverse events resulting in the total percentage being $138.4 \%$. It is also recognized that patients being treated with neuroleptic medication might have other side effects not recorded in the IC-SOHO study.

\begin{tabular}{|l|c|c|}
\hline TABLE 7: Reported Compliance to Prescribed Antipsychotic Medication (In the past \\
4 weeks for patients already undergoing antipsychotic therapy) (\%) (AMEA subset) \\
\hline & Psychiatrist & Patient \\
\hline No antipsychotic drug prescribed & 29.5 & 28.6 \\
Almost always & $45.8(64.9)$ & $51.0(71.4)$ \\
Half the time & $18.0(25.5)$ & $14.7(20.6)$ \\
Almost never & $6.8(9.6)$ & $5.7(8.0)$ \\
\hline
\end{tabular}

Compliance/adherence in the 4 weeks prior to the baseline visit was recorded from both the psychiatrist as well as the patient's point of view (Table 7). At baseline, approximately thirty percent of the psychiatrist group and the patient group reported that an antipsychotic had not been prescribed before the patient entered the study. The compliance/adherence percentages reported by the psychiatrist and patient groups followed very much the same pattern. Approximately half of the psychiatrists and the patients reported that they (the patients) almost always complied/adhered to their antipsychotic medication treatment. A limited number of patients reported that they almost never took their medication. This view was shared by their treating psychiatrists. On the other hand, approximately fourteen percent of patients reported that they took their medication half the time. This figure was higher for psychiatrists whom reported that eighteen percent of patients took their medication about half the time.

Finally, psychiatrists were requested to record the reasons for changing or initiating antipsychotic therapy at the baseline visit prior to continuing with the study (Table 8). Two thirds of the psychiatrists reported a lack of or incomplete effectiveness with previous antipsychotic medication. This was followed by intolerability to previous antipsychotic medication and a lack of or incomplete compliance/adherence with previous antipsychotic medication. Seventeen percent of patients requested a change in their medication.

TABLE 8: Reasons for Therapy Change (\%) (AMEA subset)

Loss of effectiveness $\quad 65.8$

Intolerability to previous medication $\quad 32.6$

Lack of compliance $\quad 17.4$

$\begin{array}{ll}\text { Patient request } & 17.6\end{array}$

\section{Discussion}

The IC-SOHO study is a prospective, three-year, noninterventional, observational study of schizophrenia treatment, clinical and functional characteristics. This report includes a subset of baseline results from two North African (Egypt \& Algeria) and two Middle Eastern (Turkey \& Saudi Arabia) countries. A total of 1,398 patients were enrolled from these 
four countries. The ratio of males to females as well as the mean age of patients enrolled in the study was consistent with prevalence-based samples of individuals with schizophrenia treated in outpatient facilities. ${ }^{20,25,26,27,28,29,30}$

The baseline CGI-Severity scores of the patients enrolled in the AMEA-SOHO study were generally lower than those reported in other studies. ${ }^{20,27,28}$ This might be attributable to the fact that the patients enrolled in the study consisted of non-hospitalized patients diagnosed with schizophrenia and, by definition, this group of patients would be less severely ill than those hospitalized. Other studies do not report a difference in overall symptom severity by gender.

The symptom severity for the four symptom clusters associated with schizophrenia followed the same pattern reported in similar studies. Positive and negative symptoms are usually more severe than cognitive and depressive symptoms, with the depressive symptoms the least severe. ${ }^{29}$ Negative symptoms tend to be more prevalent in males. ${ }^{26}$ Should there be a gender difference in symptom presentation, this will present an opportunity for further exploration of optimal treatment possibilities for different sexes. Patients with schizophrenia remain at risk of attempting suicide, despite being treated with antipsychotic medication with concomitant improvement in clinical symptoms.

The AMEA-SOHO sample reported here had considerable functional impairment on various levels. Although more than eighty percent of the patients were already on active antipsychotic treatment, more than half had engaged in no social activities with friends or family, and only a third of the study population was involved in a relationship. These results are slightly higher than previously reported. ${ }^{29,30}$ Only about a quarter of the AMEA-SOHO patients could care for themselves in independent living conditions, and is again consistent with other results. ${ }^{30}$ As is to be expected of a debilitating disease like schizophrenia, about three-quarters of the study group were in the care of a family member or lived in supervised residential care. Although the proportion of patients that were employed was relatively high ${ }^{29,30}$, it should be noted that this figure includes paid and unpaid employment (e.g. sheltered programs and volunteer work).

Generally, substance and alcohol dependency/abuse has been reported to be high in patients with schizophrenia. ${ }^{26}$ The fact that over ninety percent of patients in this study reported that they had never suffered from or been diagnosed with substance and alcohol dependency/abuse, might be understandable in terms of the geographical area where the study sample came from. These countries being primarily Islamic in origin might explain this finding. On the other hand this high figure might be an underestimation of the problem by the study. A further explanation of the finding can either be due to denial on the part of the patients regarding the extent of their problem, or attributable to a lack of insight into the problem as well as its dangers and consequences.

Agitation, aggression and hostility, both verbal and physical, have typically been associated with schizophrenia. ${ }^{28}$ It is therefore not surprising that almost half the patients included in the study reported this kind of behavior. Moreover, over ten percent of the patients also acknowledged that they had been compulsorily admitted, arrested or spent at least one night in jail because of this behavior.

Due to the complexity and extent of symptoms associated with schizophrenia as well as the common side effect profile of antipsychotics currently in use, schizophrenic patients are commonly prescribed adjunctive medication. ${ }^{19,20,28,31,32}$ This phenomenon was also confirmed in the AMEA-SOHO baseline data where almost half of the patients received concomitant medication and where the anticholinergics comprise the majority of those used. This high rate of anticholinergic use can be understood in the light of the high rate of typical antipsychotic usage in this sample where the old antipsychotics have been associated with a higher rate of extra-pyramidal side effects. This is in line with published data. ${ }^{19,20,28,31,32}$ The concept of 'atypicalness' has been related to a low frequency of EPS. This association needs to be further explored as the IC-SOHO study progresses. The concomitant use of anxiolytics/hypnotics, antidepressants and mood stabilizers were much less, reflecting the predefined study population (e.g. non-inclusion of schizoaffective and/or bipolar disorder). ${ }^{32}$

In contrast to the existing literature, the most frequently reported side effect in this AMEA-SOHO sample was loss of libido in almost half the study patient group. ${ }^{20,27}$ Impotence, sexual dysfunction and amenorrhoea were also reported at much higher rates than previously published. ${ }^{17,20,27}$ Although prolactin was not directly measured, several of these recorded adverse effects can indicate an elevated prolactin level. Extra-pyramidal symptoms (dystonia/akathisia/parkinsonism) have been the focus of antipsychotic treatment, and the rates reported in just over a third of patient in this study are consistent with the literature. ${ }^{19,20,28}$ Although this data does not differentiate emergent EPS between the typical and atypical groups of patients, it was expected that the atypical group of patients would have a lower incidence of reported EPS. ${ }^{18,20,27,28}$

Weight gain is an important and potentially manageable problem in patients being treated with antipsychotics. Weight gain can either be related to the disease and inherent inactivity of patients with schizophrenia, or, alternatively, to the antipsychotic medication itself. Weight gain on antipsychotics, with a possible differentiation between the various groups of drugs, has been repeatedly reported previous studies. ${ }^{28,33}$ The AMEA-SOHO results indicated that more than half of the patients at baseline were either overweight or obese. Due to the nature of the study, it was not possible to establish the exact cause of this weight gain.

Compliance/adherence is essential in the medical management of schizophrenia. The AMEA-SOHO baseline data confirms that only about half of patients treated for schizophrenia almost always complied/adhered to antipsychotic medication treatment. Good tolerance of medication is often linked to compliance/adherence. ${ }^{18}$ Medication side effects are distressing to patients and are linked to non-compliance. These side effects include extra-pyramidal side effects, neuroleptic dysphoria, akathisia, sexual dysfunction, and weight gain. ${ }^{34}$ Treatment adherence may also be improved through the use of atypical antipsychotics with few perceived side effects. ${ }^{29}$

Comprehensive and recent acceptable schizophrenia treatment guidelines recommend the use of second-generation (atypical) antipsychotics both for first-line treatment and in patients where a typical antipsychotic has failed. ${ }^{35,36}$ This was not found to be the situation at baseline in this AMEA-SOHO patient group where eighty-five percent of the patients still received either an oral or depot typical agent. This pattern of 
antipsychotic use might also be typical of countries in the developing world where there are also financial implications. It was however encouraging that a third of the patients received a newer atypical agent which might indicate a shift in prescribing patterns. At baseline, a group of patients was neuroleptic-naïve, thus providing an opportunity to study these patients.

\section{Conclusions}

Although we recognize that naturalistic observational studies may have several limitations, they could also provide new insights. Due to their nature, observational studies can be used to evaluate drug use in everyday clinical practice settings and avoid protocol-induced bias. This kind of study can provide clinicians with valuable information about the relationship between the patient, the illness and the drug, and in so doing can address the needs and concerns of both the patient and the clinician. Naturalistic studies do not however negate the need for conventional studies (RCTs) that are essential in order to establish the efficacy and safety of new chemical compounds. The IC-SOHO study thus addresses other clinical issues in order to achieve optimal clinical stability and patient satisfaction.

The baseline characteristics of the AMEA-SOHO study population presented here are from four countries in the North Africa and Middle Eastern area (Algeria, Egypt, Saudi Arabia and Turkey). To our knowledge, no similar data for this area have been previously published. Specific clinical and functional characteristics compared to antipsychotic use patterns can produce valuable short- and long-term information as a basis for the optimal treatment of schizophrenia. Specific themes discussed above have started emerging from the baseline results that will require further exploration. Ultimately, it is hoped that the complete IC-SOHO study will contribute to this knowledge base.

\section{Acknowledgements}

The authors wish to thank the IC-SOHO Study group in each specific country for their participation and contributions to the study, Linda Levitt (Leader-Scientific Information and Communications, CORI, Australia) for her review of the manuscript, as well as Andy Hodge (Senior Project Statistician, CORI, Australia) and Jason Boland (Statistical Analyst, CORI, Australia) for their assistance with the statistical analysis.

\section{References}

1. Day S. Dictionary for clinical trials. Chichester: Wiley, 1999.

2. Horwitz RI, Viscoli CM, Clemens JD, Sadock RT. Developing improved observational methods for evaluating therapeutic effectiveness. Am J Med 1990; 89(5): 630-638.

3. Sacristan JA, Soto J, Galende I, Hylan TR. A review of methodologies for assessing drug effectiveness and a new proposal: randomised database studies. Clin Therapeutics 1997; 19(6): 1510-1517.

4. Jones TC. Call for a new approach to the process of clinical trials and drug registration. B Med J 2001; 322: 920-923.

5. McKee $M$, Britton A, Black $N$, et al. Interpreting the evidence: choosing between randomised and non-randomised studies. $B$ Med J 1999; 319: 312-315.

6. Britton A, McKee M, Black N, McPherson K, Sanderson C, Bain 
C. Choosing between randomised and non-randomised studies: a systematic review. Health Technol Assess 1998; 2(13): 1-124.

7. Concato J, Shah N, Horwitz RI. Randomised, controlled trials, observational studies, and the hierarchy of research designs. N Engl J Med 2000; 342: 1887-1892.

8. Benson K, Hartz AJ. A comparison of observational studies and randomised, controlled trials. N Engl J Med 2000; 342: 1878-1886.

9. Byar DP, Simon RM, Friedewald WT, Schlesselman JJ, DeMets, $D L$, Ellenberg JH, et al. Randomized clinical trials. Perspectives on some recent ideas. N Engl J Med 1976; 295(2): 74-80.

10. Pocock SJ. Randomized trials or observational tribulations? N Engl J Med 2000; 342(25): 1907-1909.

11. Kunz R, Khan KS, Neumayer HH. Observational studies and randomised trials. N Engl J Med 2000; 343(16): 1194-1197.

12. Ioannidis JPA, Haidich AB, Lau J. Any casualties in the clash of randomised and observational evidence? B Med J 2001; 322: 879880.

13. Barton S. Which clinical studies provide the best evidence? B Med J 2000; 321: 255-256.

14. Tsuang MT. Observational versus experimental studies: Would the results be similar? Psychosom Med 1999; 61: 146-147.

15. Brambilla P, Barale F, Caverzasi E, Tognoni G, Barbui C. Clozapinetreated subjects with treatment-resistant schizophrenia: a systematic review of experimental and observational studies. Int Clin Psychopharmacol 2002; 17(4): 189-195.

16. Wehnert A. The European Post-marketing Observational Serdolect (EPOS) Project: increasing our understanding of schizophrenia therapy. Int Clin Psychopharmacol 1998; 13 (Supp/ 3): S27-30.

17. Fraile GM, Echevaria SR, Arrillaga GA, Junquera MG Risperidone in the early treatment of first-episode psychosis: a two-year follow-up study. Acta Esp Psiquiatr 2002; 30(3): 142-152.

18. Hamel B, Courtet P, Vergnes $C$, Boulenger JP. Clinical impact of atypical antipsychotics: prospective 6-month study of inpatients treated with risperidone or olanzapine. Therapie 2001; 56(6): 645652.

19. Sacristan JA, Gomez JC, Ferre F, Gascon J, Perez Bravo A, Olivares JM. Incidence of extrapyramidal symptoms during treatment with olanzapine, haloperidol and risperidone:results of an observational study. Actas Esp Psiquiatr 2001; 29(1): 25-32.

20. Sacristan JA, Gomez JC, Montejo AL, Vieta E, Gregor KJ and The EFESO Study Group. Doses of olanzapine, risperidone, and haloperidol used in clinical practice: Results of a prospective pharmacoepidemiologic study. Clin Ther 2000; 22(5): 583-599.

21. Sacristan GJC, Hernandez J, Breier A, Carrasco RP, Saiz AC, Carbonell FE. The safety of olanzapine compared with other antpsychotic drugs:results of an observational prospective study in patients with schizophrenia (EFESO Study). Pharmacoepidemiologic Study of Olanzapine in Schizophrenia. J Clin Psychiatry 2000; 61(5): 335-343.

22. Wilton LV, Heeley EL, Pickering RM, Shakir SA Comparative study of mortality rates and cardiac dysrythmias in post-marketing surveillance studies of sertindole and two other atypical antipsychotic drugs, risperidone and olanzapine. J Psychpharmacol 2001; 15(2): 120-126.

23. Williams A. EuroQol - a new facility for the measurement of health related quality of life. Health Policy 1990; 16: 199-208.

24. Rush AJ, Pincus HA, First MB, et al. Handbook of Psychiatric Measures. Washington : American Psychiatric Association, 2000.

25. Kasper S, Rosillon D, Duchesne I Risperidone Olanzapine Drug Outcomes studies in Schizophrenia (RODOS): efficacy and tolerability results of an international naturalistic study. Int Clin Psychopharmacology 2001, 16: 179-187.

26. Thornicroft $G$, Leese $M$, Tansella $M$, et al. Gender differences in living with schizophrenia. A cross-sectional European multi-site study. Schizophren Res 2002, 57: 191-200.

27. Gomez JC, Sacristan JA, Hernandez J, et al. The safety of olanzapine compared with other antipsychotic drugs: results of an observational prospective study in patients with schizophrenia (EFESO Study). J Clin Psychiatry 2000; 61(5): 335-343.

28. Alvarez E, Bobes J, Gomez JC, et al. Safety of olanzapine versus conventional antipsychotics in the treatment of patients with acute schizophrenia. A naturalistic study. Eur Neuropsychopharmacol 2003; 13: 39-48.

29. Voruganti L, Cortese L, Owyeumi L, et al. Switching from conventional to novel antipsychotic drugs: results of a prospective naturalistic study. Schizophren Res 2002; 57: 201-208.

30. Hamilton SH, Edgell ET, Revicki DA, Breier A. Functional outcomes in schizophrenia: a comparison of olanzapine and haloperidol in a European sample. Int Clin Psychopharmacol 2000; 15: 245-255.

31. Ritsneronizovsky A, Endicott J, et al. The impact of side-effects of antipsychotic agents on life satisfaction of schizophrenia patients: a naturalistic study. Eur Neuropsychopharamcol 2002; 12: 31-38.

32. Parepally H, Chakravorty S, Levine J, et al. The use of concomitant medications in psychiatric inpatients treated with either olanzapine or other antipsychotic agents. A naturalistic study at a state psychiatric hospital. Prog Neuro Psychopharmacol Biol Psychiatry 2002; 26: 437-440.

33. Rodriguez-Perez V, Lopez A, Blanco $C$, et al. Olanzapine for the treatment of chronic refractory schizophrenia: A 12-month follow-up naturalistic study. Prog Neuro-Psychopharmacol Biol Psychiatry 2002; 26:1055-1062.

34. Perkins DO. Predictors of non-compliance in patients with schizophrenia. J Clin Psychiatry 2002; 63: 1121-1128.

35. The Expert Consensus Panels. The Expert Consensus Guideline Series: Treatment of Schizophrenia 1999. J Clin Psychiatry 1999; 60(Suppl. 11): 1-82.

36. Sartorius N, Fleischhacker WW, Gjerris A, et al. The usefulness and use of second-generation antipsychotic medications Curr Opin Psychiatry 2002; 15(Suppl. 1): 1-52. 